\title{
Modeling of Thermal Processing at the Formation of Shallow Doped IC Active Regions
}

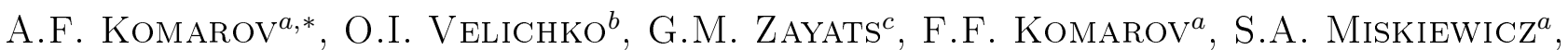
A.M. Mironov ${ }^{a}$ AND YU.V. MAKAREVICH ${ }^{a}$

${ }^{a}$ Institute of Applied Physics Problems, 7 Kurchatov St., 220108 Minsk, Belarus

${ }^{b}$ Belarussian State University of Informatics and Radioelectronics, 6 Brovki St., 220113 Minsk, Belarus

${ }^{c}$ Institute of Mathematics, Academy of Sciences of Belarus, 11 Surganov St., 220072 Minsk, Belarus

\begin{abstract}
Physical and mathematical models as well as numerical algorithms for simulation of advanced technological processes, such as thermal annealing after low-energy ion implantation used during the VLSI fabrication are presented. In this paper we propose a model that treats the migration of the impurity atoms at the thermal annealing. We take into account process nonlinearity and influence of non-uniform defects distribution as well as electric field and elastic stress on the migration of atoms. The redistribution of point defects as well as the diffusion of nonequilibrium impurity interstitials in silicon are described by time-dependent quasi-linear parabolic equations. The results of numerical calculations are presented as well.
\end{abstract}

DOI: $10.12693 /$ APhysPolA.123.804

PACS: 66.30.J-, 07.05.Tp, 07.05.Bx

\section{Introduction}

One of the ways of obtaining shallow $p-n$-junctions in the silicon-based ultra large integrated circuits (IC) is the extension of traditional technologies, namely ion implantation with the subsequent thermal processing. The fabrication of new generations of ICs is associated with the application of low-energy ion implantation and thermal annealing [1-3]. Such processes allow formation of $p-n$-junctions at the depth shallower than $30 \mathrm{~nm}$. It is significant to observe the effect of "uphill" diffusion of the impurity near the crystal surface [1, 2]. Due to high cost of IC development and fabrication, a numerical simulation that reduces the number of experimental iterations becomes extremely valuable. The necessity of obtaining adequate calculated profiles of impurity distribution implies the use of high-level kinetic diffusion models. In the present work we propose a model that treats the migration of the impurity atoms in terms of "impurity atomvacancy" and "impurity atom-interstitial atom of silicon" diffusing complexes. It also accounts for the influence of such factors as electric field, internal elastic stress and non-stationary change of defects.

\section{Diffusion model}

In accordance with modern ideas, the diffusion of substitutional impurities in silicon is carried out with the participation of point defects, namely vacancies (V) and intrinsic interstitial silicon atoms (I) which form moving impurity-vacancy and impurity-interstitial pairs with the impurity atoms (pair-diffusion mechanism) [4-7].

\footnotetext{
*corresponding author; e-mail: komaraf@bsu.by
}

Let us consider a problem of modeling the diffusion of donors or acceptors in a semiconductor. The diffusion can be described by the flux of an impurity

$$
\begin{aligned}
J & =-D^{\mathrm{E}}\left(\operatorname{grad}\left(C^{\mathrm{V}} C\right)+\frac{C^{\mathrm{V}} C}{\chi} \operatorname{grad} \chi\right) \\
& -D^{\mathrm{F}}\left(\operatorname{grad}\left(C^{\mathrm{I}} C\right)+\frac{C^{\mathrm{I}} C}{\chi} \operatorname{grad} \chi\right) \\
& +\boldsymbol{v}^{\mathrm{E}} C^{\mathrm{V}} C+\boldsymbol{v}^{\mathrm{I}} C^{\mathrm{I}} C .
\end{aligned}
$$

Here $\chi$ is the total concentration of charged particles (electrons for donors or holes for acceptors) normalized by the intrinsic concentration of electrons $n_{\mathrm{e}}$ :

$$
\chi=\frac{C-N+\sqrt{(C-N)^{2}+4 n_{\mathrm{e}}^{2}}}{2 n_{\mathrm{e}}} .
$$

In (1) and (2) the following notations were used: $C$ is the concentration of impurity in the position of substitution, $N$ is the concentration of the impurity of the opposite type (which we assume to be constant), $C^{\mathrm{V}}$ and $C^{\mathrm{I}}$ are the concentrations of vacancies and intrinsic interstitials, respectively, in neutral charge state normalized by their concentration in thermal equilibrium; $D^{\mathrm{E}}$ and $D^{\mathrm{F}}$ are the effective diffusivities of impurity atoms by means of formation, migration, and dissociation of the "impurity atom-vacancy" pairs ( $E$-centers) and formation, migration, and dissociation of the "impurity atom-silicon self-interstitial" pairs, respectively; $\boldsymbol{v}^{\mathrm{E}}$ and $\boldsymbol{v}^{\mathrm{F}}$ are the vectors of the effective drift velocity of dopant atoms in the field of elastic stresses due to migration of "impurity atom-vacancy" and "impurity atom-silicon self-interstitial" pairs, respectively. Thus, here and below the superscripts "E" and "F" refer to the diffusion mechanisms due to the interaction with vacancies and self-interstitials, respectively.

It is well known from the experimental and theoretical investigations that vacancies in silicon crystals exist 
in at least five charge states, namely, doubly negatively charged vacancies $\mathrm{V}^{2-}$, singly negatively charged vacancies $\mathrm{V}^{-}$, neutral vacancies $\mathrm{V}^{\times}$, singly positively charged vacancies $\mathrm{V}^{+}$, and doubly positively charged vacancies $\mathrm{V}^{2+}[8,9]$. Similar charge states $\mathrm{I}^{2-}, \mathrm{I}^{-}, \mathrm{I}^{\times}, \mathrm{I}^{+}$, and $\mathrm{I}^{2+}$ are also suggested for silicon self-interstitials [9]. In the simulations presented below, the temperature range of $850-1000{ }^{\circ} \mathrm{C}$ is considered. For these thermal treatments the values of $n_{\mathrm{e}}$ are within the range of $3.46 \times 10^{18}$ $8.85 \times 10^{18} \mathrm{~cm}^{-3}$. On the other hand, the characteristic concentration of cluster formation for doping with arsenic is $1.71 \times 10^{20} \mathrm{~cm}^{-3}$ and $2.54 \times 10^{20} \mathrm{~cm}^{-3}$ for the temperatures $850^{\circ} \mathrm{C}$ and $950^{\circ} \mathrm{C}$, respectively [10]. The solubility limit $C_{\text {sol }}$ for doping with boron is equal to $1.19 \times 10^{20} \mathrm{~cm}^{-3}$ for the temperature $1000^{\circ} \mathrm{C}[10]$. It means that for the case of low-energy high-dose ion implantation the value of $\chi$ is significantly greater than 1 , especially at the initial stage of annealing, and singly and doubly charged vacancies or self-interstitials can substantially influence dopant diffusion. Therefore, we describe the effective diffusivities by the following combined equation:

$$
D^{\mathrm{E}, \mathrm{F}}=D_{i}^{\mathrm{E}, \mathrm{F}} \frac{1+\beta_{1}^{\mathrm{E}, \mathrm{F}} \chi+\beta_{2}^{\mathrm{E}, \mathrm{F}} \chi^{2}}{1+\beta_{1}^{\mathrm{E}, \mathrm{F}}+\beta_{2}^{\mathrm{E}, \mathrm{F}}},
$$

where empirical coefficients $\beta_{1}^{\mathrm{E}, \mathrm{F}}$ and $\beta_{2}^{\mathrm{E}, \mathrm{F}}$ are responsible for the relative contribution of singly and doubly charged vacancies (or interstitials) to the process of impurity migration. Here $D_{i}^{\mathrm{E}}$ and $D_{i}^{\mathrm{F}}$ are the intrinsic diffusivities due to migration of "impurity atom-vacancy" and "impurity atom-silicon self-interstitial" pairs, respectively.

It is well known that local doping of silicon with a substitutionally dissolved impurity having another atomic radius compared to the host atom results in significant elastic stresses. Because the impurity distribution is changed during annealing, distribution of stresses is also a space-time function. It is assumed in Ref. [11] that the potential energy of a point defect in the field of elastic stresses $U^{\mathrm{d}}$ is proportional to the impurity concentration $C$. Then, it follows from $[12,13]$ that the effective drift velocity of point defects (including the pairs) is proportional to the gradient of the impurity concentration $C$. However, the stress calculation in the ion-implanted layers is still a very complicated problem, which requires further investigations. Indeed, the processes of point defect diffusion and cluster formation can influence the distribution of elastic stresses. Therefore, to obtain drift velocities of mobile species in the field of elastic stresses, the appropriate assumptions can be used. For example, the impurity concentration $C$ is approximated by the Gaussian function when calculating drift velocities [13].

To calculate the distributions of nonequilibrium vacancies and self-interstitials, the diffusion equations such as equations for point defects described in [14] are used. For solution of these equations, the process of defect trapping in silicon crystals is characterized by the average lifetimes of vacancies and self-interstitials. In addition, it is supposed, according to the experimental data [15], that the surface is an effective sink of silicon self-interstitials. It is also supposed that silicon self-interstitials generated during ion implantation are accumulated in the end-of-range (EOR)-defects. During thermal processing dissolution of the EOR-defects occurs that results in the increase of the concentration of self-interstitials and transient enhanced diffusion (TED) of implanted impurity. In addition, generation of silicon interstitials responsible for TED can occur due to dissolution or rearrangement of the clusters of dopant atoms [4].

According to the conservation law

$$
\operatorname{div} J+\frac{\partial C}{\partial t}=0
$$

and from (1) and (2) we obtain the following nonlinear diffusion equation:

$$
\begin{aligned}
\frac{\partial C}{\partial t} & =\sum_{i=1}^{p} \frac{\partial}{\partial x_{i}}\left(D^{\mathrm{E}}(C) \frac{\partial\left(C^{\mathrm{V}} C\right)}{\partial x_{i}}+D^{\mathrm{F}}(C) \frac{\partial\left(C^{\mathrm{I}} C\right)}{\partial x_{i}}\right. \\
+ & \left.D^{\mathrm{N}}\left(C, C^{\mathrm{V}}, C^{\mathrm{I}}\right) \frac{\partial C}{\partial x_{i}}-v_{i}^{\mathrm{E}} C^{\mathrm{V}} C-v_{i}^{\mathrm{F}} C^{\mathrm{I}} C\right), \\
p & =1,2,3, \quad 0<t \leq T,
\end{aligned}
$$

where

$$
D^{\mathrm{N}}=\frac{C\left(D^{\mathrm{E}} C^{\mathrm{V}}+D^{\mathrm{F}} C^{\mathrm{I}}\right)}{\sqrt{(C-N)^{2}+4 n_{\mathrm{e}}^{2}}} .
$$

Let us now consider Eq. (3) within the simulation domain $G$ that spans from the surface to the depth of the wafer. In the bulk of the wafer on the boundary of the domain $G$ we assume the dopant flux to be equal to zero

$$
\begin{aligned}
& D^{\mathrm{E}}(C) \frac{\partial\left(C^{\mathrm{V}} C\right)}{\partial \boldsymbol{n}}+D^{\mathrm{F}}(C) \frac{\partial\left(C^{\mathrm{I}} C\right)}{\partial \boldsymbol{n}} \\
& \quad+D^{\mathrm{N}}\left(C, C^{\mathrm{V}}, C^{\mathrm{I}}\right) \frac{\partial C}{\partial \boldsymbol{n}}-v_{\boldsymbol{n}}^{\mathrm{E}} C^{\mathrm{V}} C-v_{\boldsymbol{n}}^{\mathrm{F}} C^{\mathrm{I}} C=0,
\end{aligned}
$$

where $\boldsymbol{n}$ is the normal to the simulation boundary.

The initial conditions are of the form

$$
\left.C(\boldsymbol{x}, t)\right|_{t=0}=C_{0}(\boldsymbol{x}),
$$

where $C_{0}(\boldsymbol{x})$ is the distribution of dopant atoms after ion implantation [16].

The evolution of point defects is described by the following parabolic equation:

$$
\begin{gathered}
\frac{\partial C^{\mathrm{V}, \mathrm{I}}}{\partial t}=\sum_{i=1}^{p} \frac{\partial}{\partial x_{i}}\left(d^{\mathrm{V}, \mathrm{I}}(C) \frac{\partial C^{\mathrm{V}, \mathrm{I}}}{\partial x_{i}}+\psi_{1, i}^{\mathrm{V}, \mathrm{I}}(\boldsymbol{x}) C^{\mathrm{V}, \mathrm{I}}\right) \\
-\psi_{2}^{\mathrm{V}, \mathrm{I}}(\boldsymbol{x}) C^{\mathrm{V}, \mathrm{I}}+\psi_{3}^{\mathrm{V}, \mathrm{I}}(\boldsymbol{x}) .
\end{gathered}
$$

Here $d^{\mathrm{V}, \mathrm{I}}(C)$ is the diffusivity of point defects, $\psi_{1, i}^{\mathrm{V}, \mathrm{I}}(\boldsymbol{x})$ is the function depending on effective drift velocity in the internal elastic stress field, and $\psi_{3}^{\mathrm{V}, \mathrm{I}}(\boldsymbol{x})$ is the function dependent on the defect generation rate. The average diffusion length and the lifetime of the defects are captured in the term $\psi_{2}^{\mathrm{V}, \mathrm{I}}(\boldsymbol{x}) C^{\mathrm{V}, \mathrm{I}}$.

Initial and boundary conditions for Eq. (4) are specified as it was mentioned in [7]. In particular, boundary conditions for Eq. (4) are described as [7]: 


$$
\alpha_{1}\left(d^{\mathrm{V}, \mathrm{I}}(C) \frac{\partial C^{\mathrm{V}, \mathrm{I}}}{\partial \boldsymbol{n}}+\psi_{1} C^{\mathrm{V}, \mathrm{I}}\right)+\alpha_{2} C^{\mathrm{V}, \mathrm{I}}=\alpha_{3},
$$

where $\alpha_{1}$ denotes 0 or $1 ; \alpha_{2}$ and $\alpha_{3}$ are some constants.

An approximate solution of the nonlinear system (3), (4) is based on the splitting of differential operator of right-hand side of each of two equations. For the solution of three-dimensional problem $(p=3)$ a locally-one-dimensional method in discrete steps is used [9]. Let us introduce a uniform time grid

$$
\omega_{\tau}=\left\{t_{j}, t_{j}=\tau j, j=\overline{0, j_{0}}, \tau j_{0}=T\right\} .
$$

We assume that Eq. (3) corresponds to a chain of one-dimensional equations

$$
\begin{aligned}
& \frac{\partial C_{(k)}}{\partial t}=\frac{\partial}{\partial x_{k}}\left(D^{\mathrm{E}}\left(C_{(k)}\right) \frac{\partial\left(C_{(k)}^{\mathrm{V}} C_{(k)}\right)}{\partial x_{k}}\right. \\
& \quad+D^{\mathrm{F}}\left(C_{(k)}\right) \frac{\partial\left(C_{(k)}^{\mathrm{I}} C_{(k)}\right)}{\partial x_{k}} \\
& \quad+D^{\mathrm{N}}\left(C_{(k)}, C_{(k)}^{\mathrm{V}}, C_{(k)}^{\mathrm{I}}\right) \frac{\partial C_{(k)}}{\partial x_{k}} \\
& \left.\quad-v_{k}^{\mathrm{E}} C_{(k)}^{\mathrm{V}} C_{k}-v_{k}^{\mathrm{F}} C_{(k)}^{\mathrm{I}} C_{k}\right), \\
& k=1,2,3, t_{j-1} \leq t \leq t_{j}, \boldsymbol{x}=\left(x_{1}, x_{2}, x_{3}\right) \in G . \text { (6) }
\end{aligned}
$$

The solutions of (6) for $k=1,2,3$ are joined with the following relations:

$$
\begin{aligned}
& \left.C_{(1)}\right|_{t=t_{j}}=\left.C_{(3)}\right|_{t=t_{j}}, \quad j=\overline{1, j_{0}-1} ; \\
& \left.C_{(2)}\right|_{t=t_{j-1}}=\left.C_{(1)}\right|_{t=t_{j}}, \\
& \left.C_{(3)}\right|_{t=t_{j-1}}=\left.C_{(2)}\right|_{t=t_{j}}, \quad j=\overline{1, j_{0}} ; \\
& \left.C_{(1)}\right|_{t=0}=C_{0}(\boldsymbol{x}) .
\end{aligned}
$$

Similarly, the following system of one-dimensional equations corresponds to Eq. (4) for point defects

$$
\begin{aligned}
& \frac{\partial C_{k}^{\mathrm{V}, \mathrm{I}}}{\partial t}=\frac{\partial}{\partial x_{k}}\left(d^{\mathrm{V}, \mathrm{I}}\left(C_{k}\right) \frac{\partial C_{(k)}^{\mathrm{V}, \mathrm{I}}}{\partial x_{k}}+\psi_{1, k}^{\mathrm{V}, \mathrm{I}}(\boldsymbol{x}) C_{(k)}^{\mathrm{V}, \mathrm{I}}\right) \\
& -\psi_{2(k)}^{\mathrm{V}, \mathrm{I}}(\boldsymbol{x}) C_{(k)}^{\mathrm{V}, \mathrm{I}}+\psi_{3(k)}^{\mathrm{V}, \mathrm{I}}(\boldsymbol{x}), \\
& k=1,2,3, t_{j-1} \leq t \leq t_{j}, \boldsymbol{x}=\left(x_{1}, x_{2}, x_{3}\right) \in G .
\end{aligned}
$$

Here

$$
\sum_{k=1}^{3} \psi_{2(k)}^{\mathrm{V}, \mathrm{I}}(\boldsymbol{x})=\psi_{2}^{\mathrm{V}, \mathrm{I}}(\boldsymbol{x}), \quad \sum_{k=1}^{3} \psi_{3(k)}^{\mathrm{V}, \mathrm{I}}(\boldsymbol{x})=\psi_{3}(\boldsymbol{x}) .
$$

The values of $C_{(k)}^{\mathrm{V}, \mathrm{I}}$ for $k=1,2,3$ are joined on the time layers $t_{j}, j=\overline{0, j_{0}}$ in the same way as in (7). Equations (6) and (8) are solved sequentially for each $t=t_{j}, j=\overline{1, j_{0}}$. According to the theory of locally-one-dimensional method [9], for an approximate solution of (3) and (4) for each $t=t_{j}, j=\overline{1, j_{0}}$ we take the functions $C_{(3)} \approx C$ and $C_{(3)}^{\mathrm{V}, \mathrm{I}} \approx C^{\mathrm{V}, \mathrm{I}}$.
In order to form homogeneous conservative difference schemes for Eqs. (6) and (8), we use integro-interpolation method as described in [17].

It was shown in [18] that the long-range migration of nonequilibrium impurity interstitials is the main factor in the formation of "tails" in the region of low impurity concentration for random ion implantation into silicon crystals and implantation into preamorphized silicon layers. Accordingly, the model was improved by entering additional flux of the interstitial impurity migration as shown in [18]. An equation characterizing the inactive interstitial boron or phosphorus atoms diffusion is similar to (4). We introduced temperature-dependent activation time [19] after which the impurity atoms are considered as electrically active, and their further diffusion is calculated using Eq. (3).

\section{Calculation results}

A software package designed on the basis of the described models was integrated into the Silvaco ATHENA Process Simulation Framework. It enables the use of our models and calculation methods in designing flow solutions alternative to implemented in the well-known TCAD software products, mainly in solving the problems with the shallow depth of formed doped regions. The developed software was used to simulate various processes, including low energy ion implantation of $\mathrm{B}, \mathrm{BF}_{2}, \mathrm{P}, \mathrm{As}$, $\mathrm{Sb}, \mathrm{C}$ in the silicon structure, corresponding to typical heavily doped small active areas of VLSI components, and subsequent thermal annealing. The simulation results agree well with the experimental data obtained by means of secondary ion mass spectroscopy (SIMS) and spreading resistance profiling, including possibility to predict experimentally observable extended "tail" formation in the region of low impurity concentration, "kink and tail" profile shapes, the "uphill" diffusion and local maxima formation at a crystal surface. The models are computationally effective and applicable to simulation of one- and two-dimensional impurity concentration profiles in multilayered semiconductor structures.

We simulated various experiments described in the literature and compared the calculated data with the empirical results. In this section we will describe two such experiments. In Figs. 1 and 2, the simulation results for very low energy ion implantation at 2 and $5 \mathrm{keV}$, respectively, for boron [20] and arsenic [2], followed by thermal annealing are compared to the experimental data.

In Fig. 1, the SIMS data from Ref. [20] and our simulation results are presented for $1 \times 10^{14} \mathrm{~cm}^{-2}$ boron implanted in silicon with an energy of $2 \mathrm{keV}$ and then annealed for $10 \mathrm{~s}$ at a temperature of $1000^{\circ} \mathrm{C}$. It is assumed that the contribution of vacancies may be neglected because the self-interstitial supersaturation is over $10^{4}$ [21], and hence the contribution of self-interstitials is much larger than that of vacancies.

The boron diffusion occurs through the formation, migration and dissociation of mobile self-interstitial dopant 


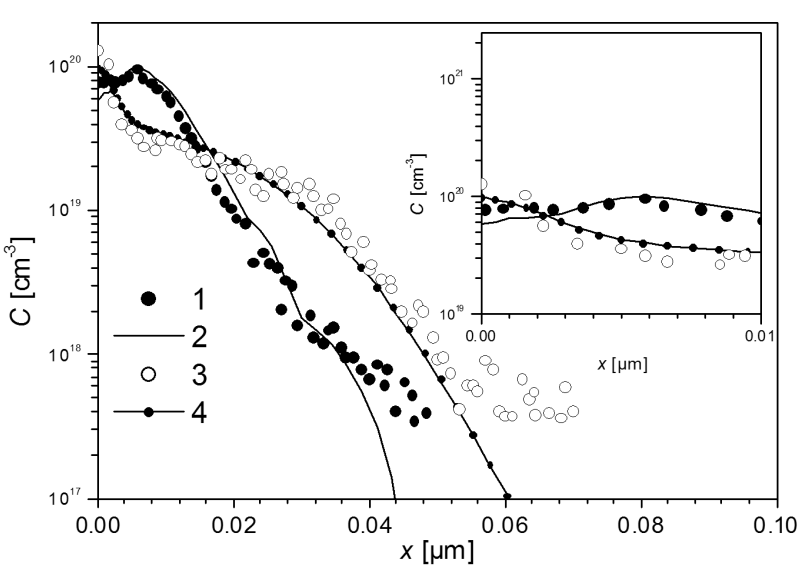

Fig. 1. Results of our simulation (4) compared to the experimental SIMS profile (3) from Ref. [12] for $1 \times 10^{14} \mathrm{~cm}^{-2}$ boron implanted in silicon with an energy of $2 \mathrm{keV}$ and annealed for $10 \mathrm{~s}$ at a temperature of $1000{ }^{\circ} \mathrm{C}$. (1) and (2) are the implanted boron profiles (SIMS and simulated, respectively).

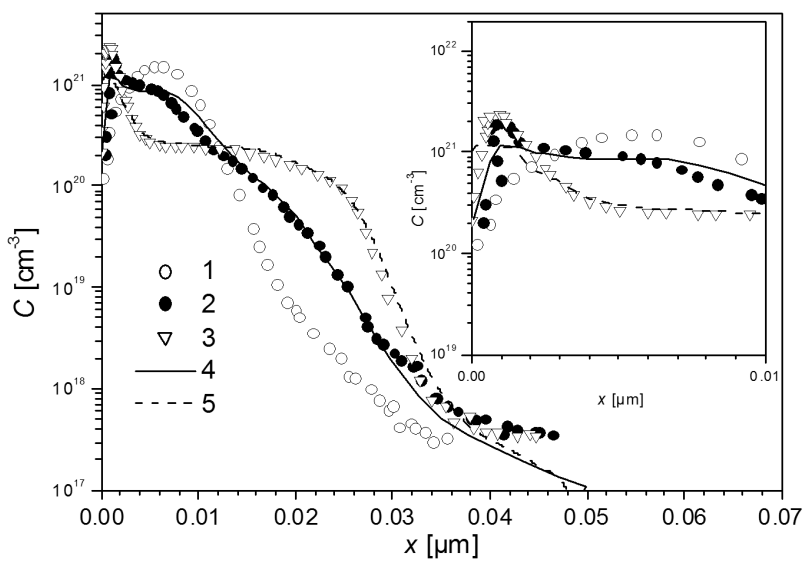

Fig. 2. Simulation results compared to the experimental ToF-SIMS profiles from [2] for $5 \mathrm{keV}$ arsenic implanted at a dose of $1 \times 10^{15} \mathrm{~cm}^{-2}$ in silicon and then annealed (RTA). (1), (2) and (3) are the experimental profiles of as-implanted and annealed at 850 and $950{ }^{\circ} \mathrm{C}$ arsenic; (4) and (5) are our simulation results for 850 and $950^{\circ} \mathrm{C}$ RTA, respectively.

pairs. The effect of electrical field is also taken into account. The essential simulation parameters for diffusivity evolution are $\beta_{1}^{\mathrm{F}}=0.7 ; \beta_{2}^{\mathrm{F}}=0$. In the boundary conditions for defects (self-interstitials) at the silicon surface in Eq. (5), the parameters $\alpha_{1}=1, \alpha_{2}=10^{14}$, and $\alpha_{3}=0$ are used.

In Fig. 2, the experimental results of Ref. [2] are compared with the simulation data. To model diffusion of As atoms, a few simplifications were done. It is assumed that these atoms coupled with self-interstitials diffuse mainly on interstitials. Thus, the effective diffusivity observed represents the diffusivity of these mobile pairs multiplied by the fraction of dopants which are paired.
Self-interstitial drift in the field of electric stresses was neglected. The essential simulation parameters in this case are $\beta_{1}^{\mathrm{F}}=3.67 ; \beta_{2}^{\mathrm{F}}=1.34$. In the boundary conditions for point defects at the silicon surface, the parameters $\alpha_{1}=1, \alpha_{2}=10^{14}$, and $\alpha_{3}=10^{-9}$ are used.

In Ref. [2], arsenic ion implantation at the energies of 5,10 and $15 \mathrm{keV}$ at a high dose of $1 \times 10^{15} \mathrm{~cm}^{-2}$ was carried out into Si wafers at room temperature at a tilt angle of 7 degree. After ion implantation, the wafers were subjected to rapid thermal annealing (RTA) in a tungsten-halogen lamp system, in nitrogen ambient. The RTA conditions were: temperature $650-950{ }^{\circ} \mathrm{C}$ and time 10-30 s. Time-of-flight SIMS arsenic concentration depth profiling has been performed. Our results were found to be in a good agreement with the ToF-SIMS profiles of annealed samples. In Fig. 2, we show the results for $5 \mathrm{keV}$ implantation followed by RTA at $850 \mathrm{ad} 950^{\circ} \mathrm{C}$.

Therefore, the results of the simulations fit the experimental data very well, which implies that the proposed model can be used to predict the results of experiments.

\section{Conclusion}

A model of high concentration transient enhanced diffusion of impurity atoms implanted into Si has been developed. The model takes into account process nonlinearity and influence of non-uniform defects distribution as well as electric field and elastic stress on the migration of atoms. The redistribution of point defects as well as the diffusion of nonequilibrium impurity interstitials in silicon are described by time-dependent quasi-linear parabolic equations. These features make it possible to use the model for simulating the low-energy-implanted dopant diffusion during thermal annealing. A software package designed on the basis of the described model enables the use of new models and calculation methods in designing flow solutions alternative to implemented in the well-known TCAD software products, mainly in solving the problems with the shallow depth of formed doped regions.

\section{Acknowledgments}

This work was partially supported by the Belarussian Republican Foundation for Fundamental Research (grant T11-159).

\section{References}

[1] S. Solmi, M. Ferri, M. Bersani, D. Giubertoni, V. Soncini, J. Appl. Phys. 94, 4950 (2003).

[2] D. Girginoudi, N. Georgoulas, A. Thanailakis, E.K. Polychroniadis, Mater. Sci. Eng. B 114-115, 381 (2004).

[3] A. Ruffell, I.V. Mitchell, P.J. Simpson, J. Appl. Phys. 97, 123518 (2005).

[4] F.F. Komarov, O.I. Velichko, V.A. Dobrushkin, A.M. Mironov, Phys. Rev. B 74, 035205 (2006). 
[5] A.K. Fedotov, O.I. Velichko, V.A. Dobrushkin, J. Alloys Comp. 382, 283 (2004).

[6] F.F. Komarov, O.I. Velichko, A.M. Mironov, V.A. Tsurko, G.M. Zayats, Proc. SPIE 6260, 566 (2006).

[7] B.B. Khina, Def. Diff. Forum 277, 103 (2008).

[8] R.B. Fair, in Silicon Integrated Circuits, Pt. B, Ed. Kahng Dawon, Academic Press, New York 1981, p. 1.

[9] P. Pichler, in: Computational Microelectronics, Ed. S. Selberherr, Springer, Wien 2004.

[10] S. Solmi, in: Encyclopedia of Materials: Science and Technology, Eds. K.H.J. Buschow, R.W. Cahn, M.C. Flemings, B. Ilschner, E.J. Kramer, S. Mahajan, P. Veyssière, Elsevier Sci., 2001, p. 2331.

[11] J. Pezoldt, P.V. Rybin, D.V. Kulikov, Yu.V. Trushin, R.A. Yankova, M. Voelskow, U. Kreissig, Nucl. Instrum. Methods Phys. Res. B 166/167, 758 (2000).

[12] O.I. Velichko, A.K. Fedotov, Mater. Sci. Eng. B 99, 567 (2003).

[13] O.I. Velichko, Appl. Math. Mod. 35, 1134 (2011).
[14] O.I. Velichko, Yu.P. Shaman, A.K. Fedotov, A.V. Masanik, Comput. Mater. Sci. 43, 279 (2008).

[15] Y. Lamrani, F. Cristiano, B. Colombeau, E. Scheid, P. Calvo, H. Schäfer, A. Claverie, Nucl. Instrum. Meth. Phys. Res. B 216, 281 (2004).

[16] A.F. Burenkov, F.F. Komarov, M.A. Kumakhov, Tables of Ion Implantation Spatial Distribution, Gordon and Breach, New York 1986.

[17] A.A. Samarskii, Theory of Difference Schemes, Nauka, Moscow 1977.

[18] O.I. Velichko, A.P. Kavaliova, Physica B 407, 2176 (2012).

[19] E. Schroer, V. Privitera, F. Priolo, E. Napolitani, A. Carnera, Mater. Sci. Semicond. Proc. 3, 303 (2000).

[20] F. Boucard, F. Roger, I. Chakarov, V. Zhuk, M. Temkin, X. Montagner, E. Guichard, D. Mathiot, Mater. Sci. Eng. B 124-125, 409 (2005).

[21] S. Solmi, F. Baruffaldi, R. Canteri, J. Appl. Phys. 69, 2135 (1991). 